\title{
CONTINGENCY CONTRACTING TO ENHANCE PATIENT COMPLIANCE: A REVIEW
}

NANCY K. JANZ, R.N., M.S. and MARSHALL H. BECKER, Ph.D., M.P.H.

Department of Health Behavior and Health Education, School of Public Health, University of Michigan, Ann Arbor, Michigan

PAUla E. haRTMAN, Ph.D.

Department of Surgery, College of Medicine,

Northeastern Ohio University, Rootstown, Ohio

\begin{abstract}
Researchers and practitioners have expressed considerable interest in contingency contracting as a promising intervention strategy for enlisting patient cooperation, particularly with regard to long-term treatment regimens. After brief examination of the theoretical background, the authors summarize advantages of contracting, describe elements essential to the development of a contract, and enumerate those ingredients in the contracting process thought to be critical for achieving optimal results. They review relevant research efforts in terms of their designs, methods, target and contracted health-related behaviors, contingencies employed, and initial and follow-up results. On the basis of this review, current issues regarding contingency contracting are raised, and practical considerations for large-scale application are noted along with recommendations for future research.
\end{abstract}

\section{INTRODUCTION}

Patient noncompliance continues to be a serious obstacle to the achievement of treatment goals. At a time when efficacious therapies are available for many illnesses, reported estimates of noncompliance (typically ranging from $30 \%$ to $60 \%$ ) are discouraging. ${ }^{1}$ Some authorities consider the problem of enlisting patient cooperation the most serious challenge facing medical practice today. ${ }^{2}$ Noncompliance adversely affects both the quality and costs of medical care by disrupting or negating the potential benefits of treatment; involving the patient in additional, unnecessary diagnostic procedures, treatments, and visits; and interfering with the clinician-client relationship.

A new and promising tool for enhancing cooperation is the contingency contract. The contract capitalizes on the client-provider relationship by actively involving the patient in the therapeutic decision-making process and providing additional incentives (reinforcers) for achieving treatment objectives. This paper will describe the elements of contingency contracts and the contracting process and will review investigations using contracts to enhance compliance.

\section{THE CONTINGENCY CONTRACT}

A contingency contract is a specific negotiated agreement that provides for the delivery of positive consequences or reinforcers contingent upon desirable behavior (and sometimes the delivery of negative consequences when undesirable behaviors are displayed). Lewis and Michnich ${ }^{3}$ view these contracts as "negotiated agreements between the parties as to the relative and absolute authority and responsibility of each in achieving a defined goal or objective that is mutually decided upon by both.",

Contingency contracting has its theoretical roots in operant or instrumental conditioning. Operant procedures, based most notably on the work of Skinner, emphasize the importance of exploring the consequences (rather than the causes) of behavior. Skinner demonstrated that consequences of behavior (reinforcing stimuli) can determine the pattern of subsequent behavior. ${ }^{5}$ Positive consequences can be used to increase the frequency of desired behavior, while negative or aversive consequences can be used to decrease the frequency of undesirable behavior.

Little has been published concerning the theoretical basis for using a written contract to implement contingency management. In the $1960 \mathrm{~s}$, the term "contract" was frequently linked with approaches in counseling and psychological intervention. For example, Sulzer ${ }^{6}$ described a "psychotherapeutic contract"' as a 
jointly agreed-upon and always modifiable explicit set of guidelines for the relationship between the patient and the therapist. Contingency contracting was later applied in educational settings between teachers and students. ${ }^{7,8}$ Some position papers have urged the use of a contract between patients and physicians as a means of enhancing the therapeutic relationship and promoting patient compliance. ${ }^{9}$

Lewis and Michnich ${ }^{3}$ note that the contracting process clarifies the relative responsibilities of both provider and consumer through the explicit exchange of information. This, in turn, creates a perceived or real transfer of power from the provider to the client. However, the relative contributions of the various points of impact in contingency contracting as an intervention strategy have not been clearly delineated. Thus reported successes may be more a function of the process inherent in the formation of a contract than of the written contract itself. On the other hand, Kanfer ${ }^{10}$ argues that the tendency of members of society to fulfill obligations accepted in formal agreements enhances motivation for behavior change. From a self-regulation perspective, Kanfer suggests that the contract adds power over a private resolution or vague intention to change behavior by establishing reinforcing consequences and by relinquishing partial control to another individual.

\section{ELEMENTS OF THE}

\section{CONTINGENCY CONTRACT}

A number of elements have been identified that are considered desirable in the development of a good contingency contract:

1. The agreed-upon (negotiated) goal to be accomplished should be clearly and specifically described. ${ }^{3}$

2. The precise responsibilities (behaviors) that each party in the contract has in achieving the goal must be detailed, $3,11,12$ including the setting of time or frequency requirements for performance of the behaviors. ${ }^{3,12}$

3. The required behaviors should be easily observed, measured, and recorded to facilitate reinforcement decisions. ${ }^{12,13}$

4. There must be clear specification of the positive reinforcements to be received contingent upon achievement of the specific behaviors. ${ }^{11,12}$ We would suggest that the timing for delivery of the reinforcements also be specified.

5. A detailed description of what will be done if any party fails to fulfill his/her responsibilities under the terms of the contract must be provided ${ }^{13}$; this may include some aversive consequences or sanctions. ${ }^{11,12}$
6. A "bonus clause" may be included, which specifies additional rewards if the client exceeds his/her specific responsibilities. ${ }^{11,12}$

7. Specific dates for contract initiation, termination, and negotiation/renewal should be included.

8. The contract should be signed by all parties involved. ${ }^{3}$

An example of a contingency contract appears in the Appendix.

Proponents of the use of contracting to obtain client cooperation with health-related recommendations have advanced the following arguments in its favor: (1) the client becomes a participant in the decision-making process regarding the treatment plan and makes a commitment to behavior change; (2) an opportunity is provided to discuss potential problems and solutions; (3) the contract fosters accountability through written specification of each party's share of the responsibility for the client's health care; (4) the signatures of all parties involved create formal commitment; (5) the document provides an instrument of communication for others involved in the client's care and facilitates evaluation of progress by permitting comparison of activities and outcomes with the precise terms of the contract; (6) the contingency component provides additional incentive through reinforcement of the desired behaviors.

\section{THE CONTRACTING PROCESS}

There is general agreement that several aspects of the contracting process are critical to achieving success: (1) the client must be actively involved in the selection of both behaviors and reinforcers; (2) each element of the contract must be accepted fully by all relevant parties; (3) complex behaviors should be broken down into small, achievable components (or successive approximations) that progressively move the client toward the ultimate goal, and each small step should be reinforced; (4) the contract should be modifiable by negotiation among the parties involved. Some authors have suggested the value of collecting baseline data on the presence or absence of the desired behavior. ${ }^{13}$ Others recommend that behaviors be rehearsed before commitment by the client. ${ }^{12}$

Steckel ${ }^{13}$ advocates specification of the target behavior in measurable terms, continuous reinforcement when the behavior is first undertaken, and gradual removal of the reinforcement as the desired behavior becomes established (fading). Kanfer ${ }^{12}$ notes that the timing for delivery of reinforcement contingencies should be arranged to follow the response as quickly as possible, and Melamed and Siegel ${ }^{14}$ recommend that the value of the reinforcer be consistent with the effort required by the behavior. 


\section{STUDIES EVALUATING CONTINGENCY CONTRACTING TO INCREASE COMPLIANCE}

The studies to be discussed are presented in Table I, and are grouped by area of target behavior.

\section{Weight Change}

Using a randomized control design, Harris and Bruner ${ }^{15}$ attempted to obtain weight loss in obese adults with a contingency contract that included the forfeit of relatively small amounts of deposited money for failure to attain the goal. Results achieved by the contract clients were compared with those from a self-control intervention and a placebo condition. Short-run assessment of the intervention strategies revealed that contracting resulted in a mean weight loss almost twice that obtained with self-control methods; furthermore, client dropout from the education-attention control group was so great as to render findings for that group invalid. However, at the ten-month follow-up (without additional intervention), both the self-control and contracting groups evidenced substantial recidivism.

Aragona and colleagues ${ }^{16}$ compared the efficacy of two weight-reduction programs for obese girls with each other and with a no-treatment control group. Both the "response-cost with reinforcement," and "responsecost only" interventions involved contracts that included the clients, their parents, and the therapist. The contracts required the parents to deposit money, which would be forfeited if their child's weight-loss objectives were not reached, to graph calorie intake and weight, to keep a food-intake diary, and to attend group meetings. The parents of children in the reinforcement group had the additionally contracted obligation to provide daily rewards (negotiated with the child and the experimenter) for adherence to the weight-loss regimen. These reinforcers might be praise, tokens, money, or the like. Findings for the initial 12-week study period indicated significant weight loss in both treatment groups, compared with slight weight gain for the control group. The group with reinforcement showed the best results. Unfortunately, treatment effects began to fade by the eight-week follow-up and had completely disappeared at 31 weeks. (Indeed, the reinforcement group had returned to baseline weight, while the remaining treatment group and the control group had experienced substantial weight gain.)

A more unusual and dramatic case of contingency contracting for weight loss involved eight subjects who agreed to lose at least 25 pounds and to risk forfeiture of any or all of a number of valuable personal items relinquished at the onset of treatment. Mann ${ }^{17}$ used a single-subject reversal design wherein, for a time, each subject (following the experimental condition) experienced a "control" period during which weight loss was expected but the contracted contingencies were not in effect. Deposited valuables could be returned under three conditions: immediate (for each two-pound loss from baseline); two-week (for reaching the previously contracted two-week goal); and terminal (for reaching the final weight goal, in which case all remaining valuables were returned). A gain of two pounds or failure to achieve contracted two-week or terminal objectives resulted in loss of valuables. Data at 16 weeks showed a mean weight loss of 32 pounds. Moreover, while the experimental condition yielded an average loss of 1.7 pounds/week, the reversal ("no contingencies") condition experienced an average weight gain of 1.4 pounds/week. However, Mann notes that "anecdotal reports from some of the subjects indicated that they had used extreme measures . . . to lose weight rapidly and temporarily in order to avoid aversive consequences." No long-term follow-up was conducted.

One investigation employed contingency contracting to achieve weight gain. Agras and Werne ${ }^{18}$ used both positive reinforcers and punishment with a group of 25 female inpatients suffering from anorexia nervosa. In addition to receiving an extensive program of behavioral treatments (such as ongoing psychotherapy or assertiveness and relaxation training), all subjects negotiated contingency contracts whereby weight gain was rewarded with telephone, visitor, and freedom-ofmovement privileges, while weight loss was penalized by isolation and forfeiture of privileges. Weight gain beyond contracted objectives eamed such bonuses as additional physical therapy and home visits.

The investigators reported a mean group increase of $20 \%$ above pretreatment weight; however: (1) the measurement period fluctuated greatly across subjects, ranging between 12 and 113 days; (2) three subjects dropped out of the program and were therefore viewed as treatment failures; and (3) there was no control group, so one cannot determine how much of the weight gain was due to the contingency contract as opposed to other interventions supplied by the overall behavioral treatment program. No long-term follow-up was reported.

Another study utilizing powerful contingencies $\mathbf{( \$ 2 0 0}$ deposit) in an effort to encourage weight loss among severely obese adults was conducted by Jeffery and coworkers. ${ }^{19}$ Study participants were randomly assigned to one of three treatment contract conditions in which increments of $\$ 20 /$ week could be earned back for weight loss of two pounds/week or two pounds below the lowest previous weight (weight-contract condition), selfreported changes in caloric intake (calorie-contract group), or attendance at the educational group meetings (attendance-contract condition). Money not earned back by fulfilling contract conditions was permanently forfeited. A no-contract control group was formed from individuals who were unwilling to participate in the contract conditions. All participants were involved in the 


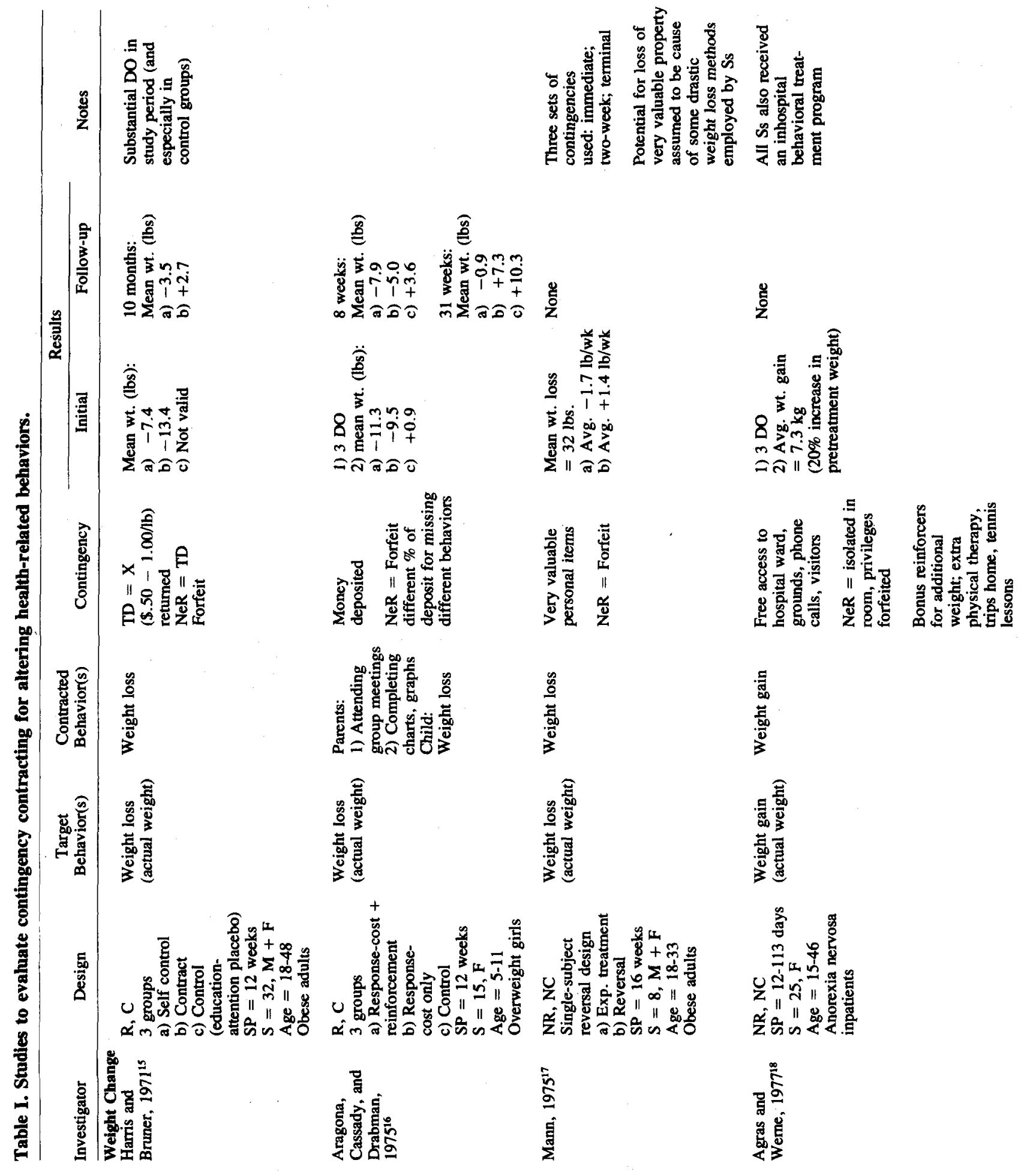




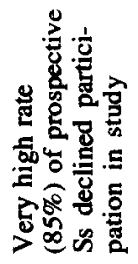

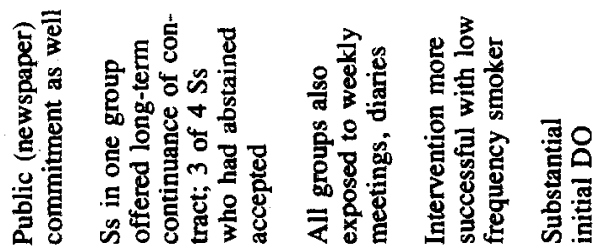

莡

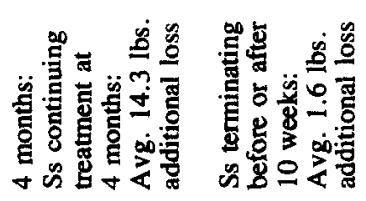

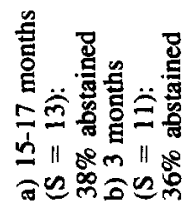

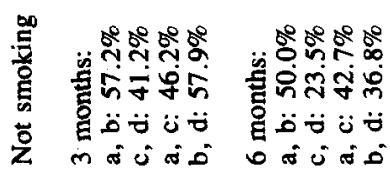

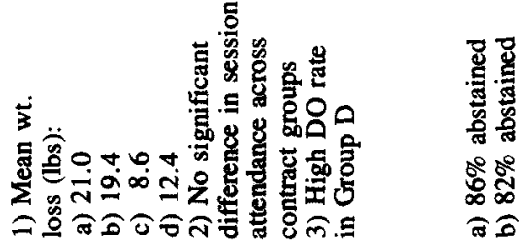

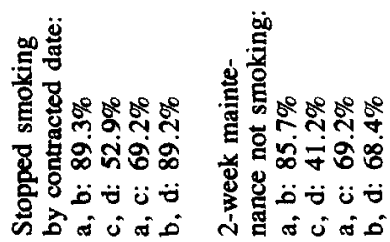

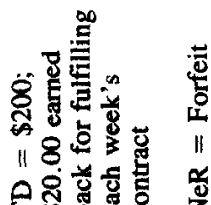

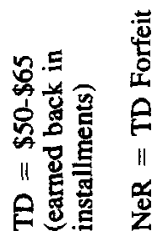

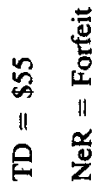

尊

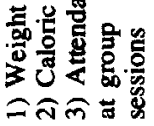

营兽

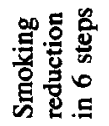

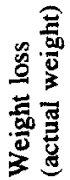

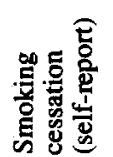

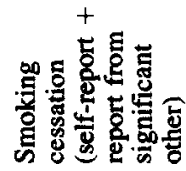

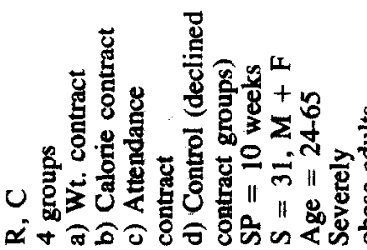
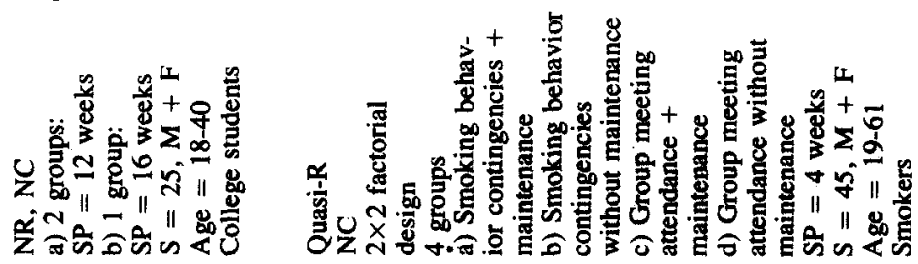

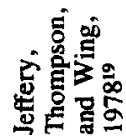

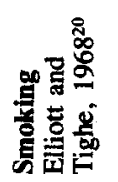

$\frac{\tilde{\sigma}}{\tilde{\sigma}}$ 


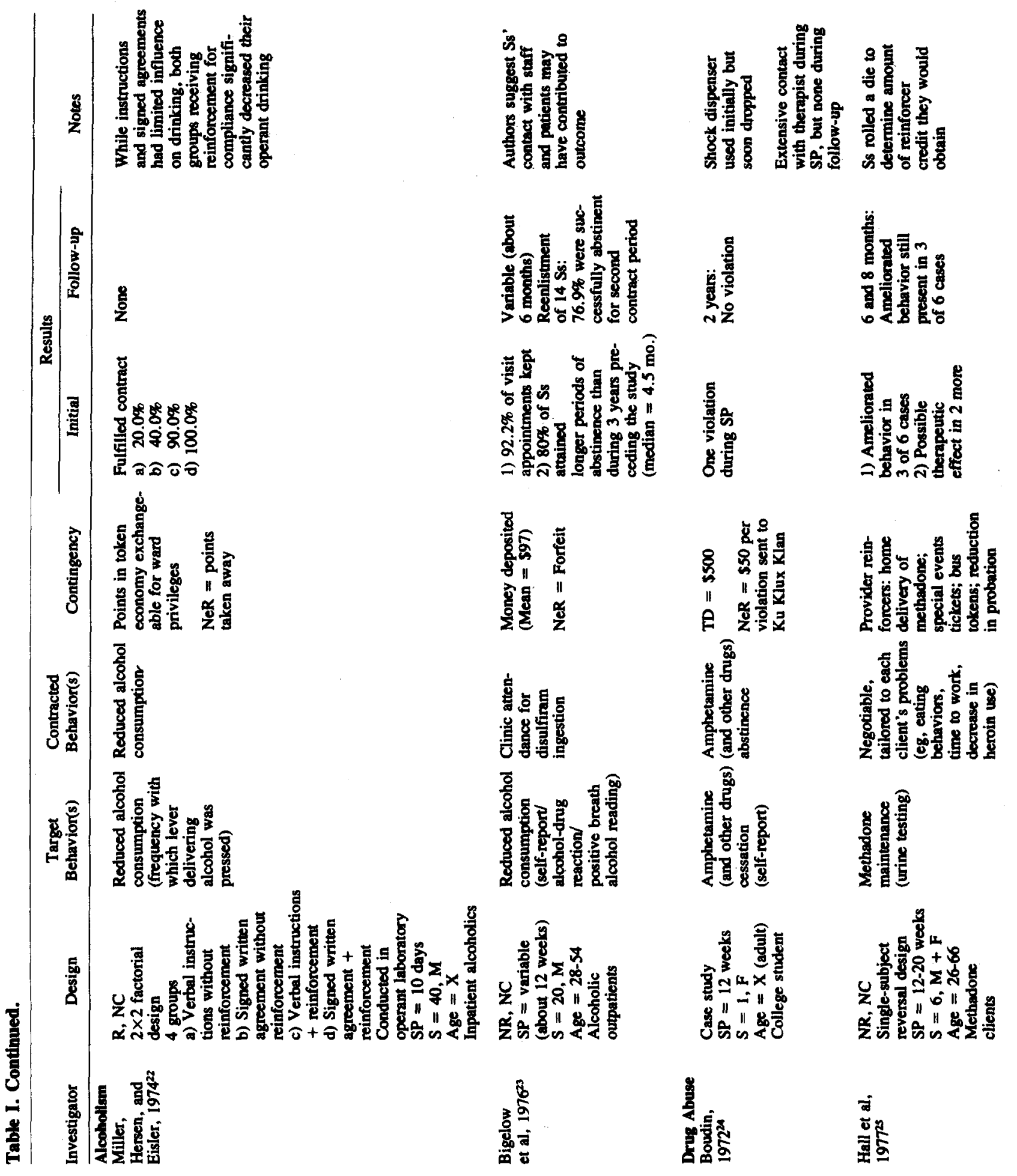




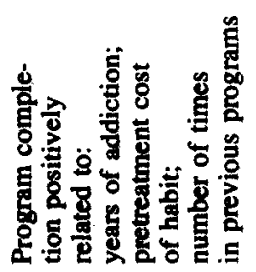

z

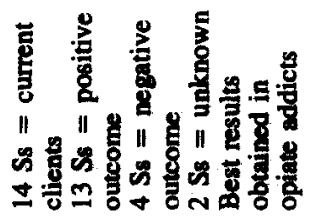

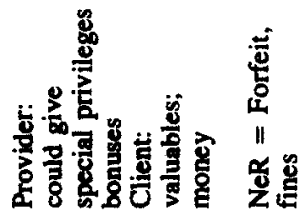

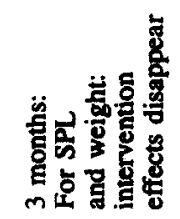

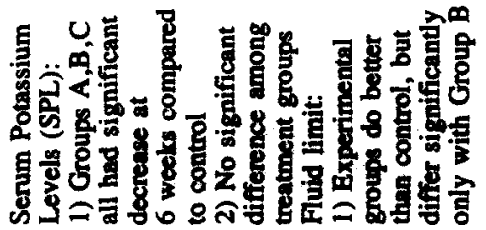

$\frac{8}{3}$

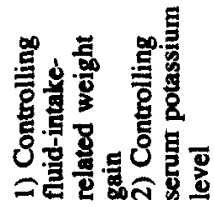

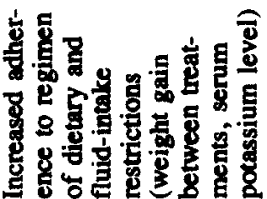

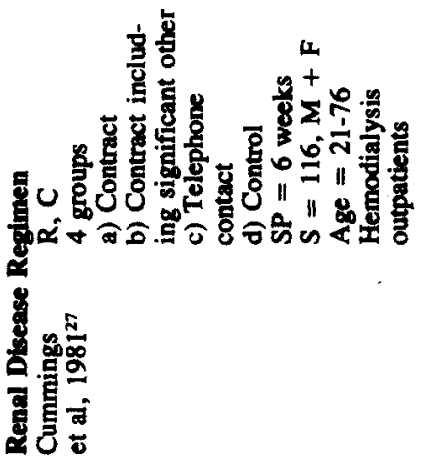




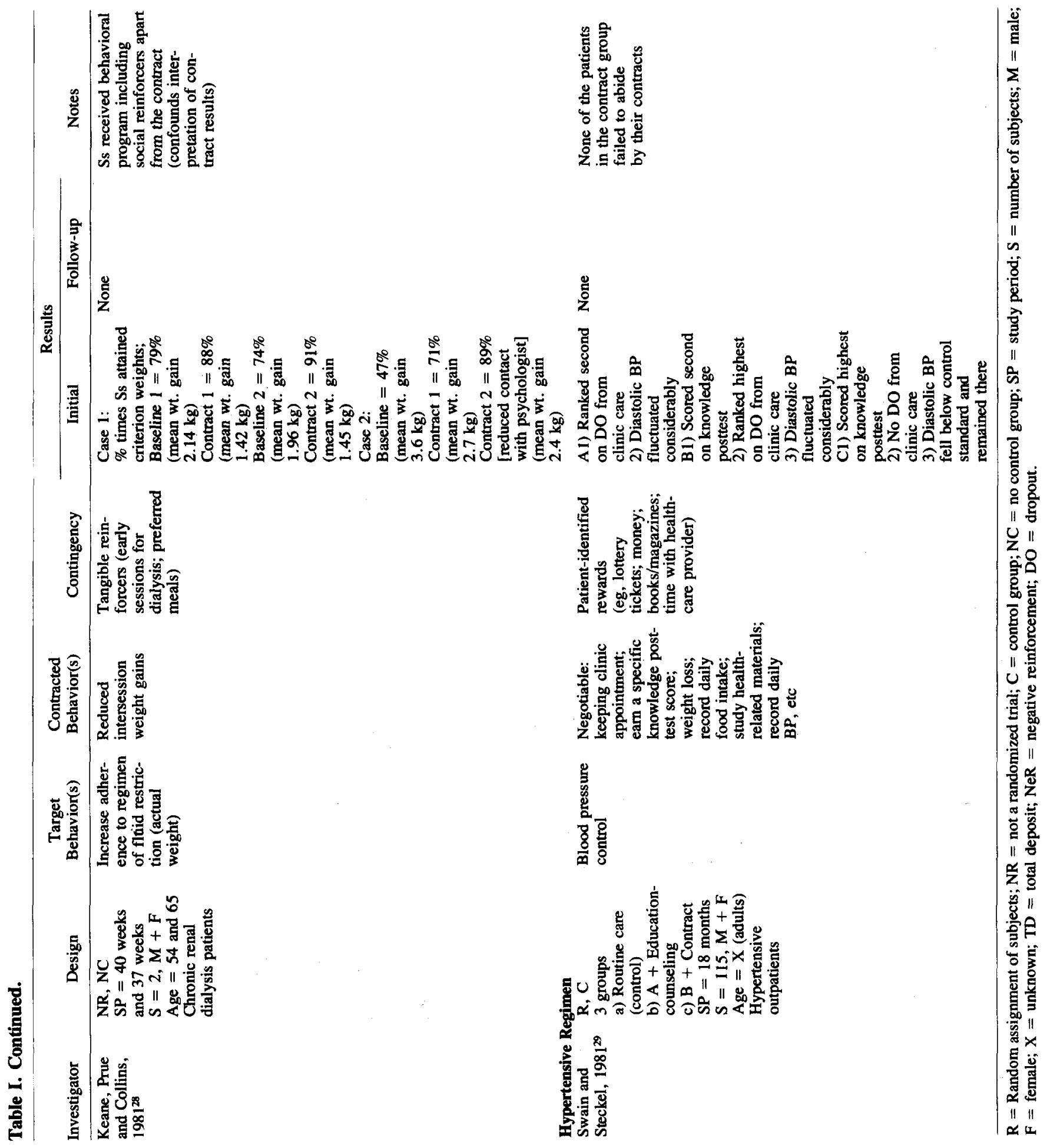


weekly behavior modification and educational sessions, that is, all subjects were expected to attend meetings, keep caloric-intake diaries, lose two pounds/week, and so forth, but they could only earn back portions of the deposit by successful achievement of objectives specified by their contract condition.

At the end of the ten-week study period, all groups had attained a significant mean weight loss, with the weight-contract and calorie-contract groups losing substantially more than the attendance-contract or control group. Moreover, in contrast to Mann's ${ }^{17}$ study, there was no evidence that participants resorted to unhealthy eating habits or drastic measures to lose weight.

Sixteen of the 31 original participants elected to continue in a follow-up program that involved writing their own contracts at monthly intervals for either caloric restriction or weight loss. Results obtained four months after the end of the ten-week study indicated that subjects who had continued under contract conditions lost considerably more weight than did those who chose to terminate their involvement at or before ten weeks.

\section{Smoking}

Elliott and Tighe ${ }^{20}$ used contingency contracting to assist a group of self-directed college-age volunteers to stop smoking. All study participants relinquished money ( $\$ 50$ to $\$ 65$ ), which was immediately forfeited if they used tobacco of any kind during the study period. A shaping procedure was used in which various amounts of money could be earned back for successively longer periods of abstinence. At the initial meeting, volunteers were exposed to a lecture on the health hazards of smoking and on management of common problems encountered in the early phases of quitting. Two additional provisions of the contract required all subjects to read articles on smoking provided by the investigators and to agree to a personal public commitment to stop smoking (name published in the college newspaper as a smoking-cessation program participant).

Three small groups, two nunning for 12 weeks and one for 16 weeks, resulted in a total sample of 25 . The investigators report an impressive overall short-run success rate of $84 \%$ remaining abstinent. However, at the three-month follow-up, the 16-week group's abstinence level had declined to $36 \%$, and at the 15 - to 17 -month follow-up, the 12-week groups' abstinence level had dropped to $38 \%$. Thus recidivism was quite high in the long run.

Because the study contained no control group, it is difficult to assess the relative contribution to smoking cessation of the various elements of the contract (such as total deposit versus public commitment versus literature on health hazards of smoking). Furthermore, the subjects were highly motivated volunteers, all of whom received an initial antismoking lecture and there- fore might have done as well without the contracting intervention. The investigators, however, did report that three fourths of the subjects gave "fear of losing money" as a major factor in remaining abstinent.

Winett ${ }^{21}$ investigated the efficacy of a six-step program in which the designated times when individuals were permitted to smoke were gradually eliminated in a hierarchical fashion. Forty-five adult smokers deposited $\$ 55$ at entry into the four-week study period. A $2 \times 2$ factorial design was used to determine the effect of returning portions of the deposit contingent on altered smoking behaviors as opposed to attendance at group meetings, as well as the effects of including or excluding a two-week maintenance period of smoking as part of the contracted behavior. In the "smokingbehavior contingent"' groups, various portions of the deposit were returned for adherence to the established time schedules, for reducing baseline smoking by $80 \%$, and, ultimately, for stopping. The "attendance" groups earned back their deposit by attending weekly group meetings. The "maintenance" groups were to continue their commitments (ie, smoking cessation or meeting attendance for two weeks beyond the stop-smoking point), and the "no maintenance"' groups were required only to report on their smoking behavior.

Each participant received a manual explaining the rationale and procedures of the program and a pocket notebook to record the time of each cigarette smoked. All subjects were also expected to attend weekly group meetings on smoking and health. A final element of the study required each participant to identify four significant others (two outside the nuclear family) who could be contacted at some point during the study period to verify the subject's smoking behavior.

Initial study results revealed that $89 \%$ of the "smoking-behavior contingent", groups had successfully quit by their contracted dates, compared with only $53 \%$ of the "attendance" groups. At two-week follow-up, the results were $86 \%$ and $41 \%$, respectively. Initial cessation rates were $69 \%$ for the "maintenance", groups and $84 \%$ for the "no maintenance" groups. There was no significant difference between these groups at the two-week follow-up. The abstinence rate at three months was $51 \%$ and declined to $40 \%$ at six months.

Responses to a follow-up questionnaire mailed to the study participants at the end of the program indicated that the contract and commitment (of deposit) were rated as more helpful than approaches utilizing information received, recording procedures, plans for reducing and stopping, and group meetings. The contracting procedure was more successful with smokers who smoked fewer than 23 cigarettes a day. Finally, Winett ${ }^{21}$ suggests that future programs planning to involve significant others should fully incorporate them into the contractual agreements. 


\section{Alcoholism}

In an operant laboratory setting (where by pressing a lever subjects obtained alcohol), Miller and associates ${ }^{22}$ used a $2 \times 2$ factorial design to evaluate the effects of different components of contracting on the alcohol consumption level of inpatient alcoholics. Specifically, the investigators compared verbal instructions with written and signed instructions, varying the presence of reinforcement. The target behavior was a reduction in alcohol consumption to a level of no more than $50 \%$ of the subject's mean consumption determined at four pretest sessions. For the two reinforcement groups, achievement of the target goal earned points exchangeable for ward privileges; failure resulted in the loss of twice as many points as would have been earned.

After four sessions, results indicated that the "reinforcement component" of the contract had the most powerful influence on behavior $(90 \%$ to $100 \%$ fulfilled their contractual obligations); signed, written instructions achieved $40 \%$ compliance, while verbal instructions alone were successful in only $20 \%$. A confounding variable influencing interpretation of these findings was the fact that subjects in the "reinforcement" groups necessarily received feedback concerning whether or not they had attained their goals; such information may well have contributed to the observed outcomes. No longterm follow-up was conducted.

An outpatient treatment program provided the setting for Bigelow and associates ${ }^{23}$ to evaluate the usefulness of contingency contracting in maintaining regular disulfiram ingestion among problem drinkers. The investigators report on 20 male volunteers whose initial deposits (mean of $\$ 97$ ) were sacrificed in increments of $\$ 5$ to $\$ 10$ for failure to report to the clinic for their disulfiram dose. Any remaining money would be returned to the participant at the end of the contract period. Each visit to the clinic resulted in not only contact with the nursing staff to receive disulfiram but also an opportunity to visit socially with the staff and with alcoholic inpatients. Although the contract contingencies focused on clinic visitation rather than on drinking behavior, the frequency of visits allowed for careful validation of each patient's drinking status.

After approximately 12 weeks, $80 \%$ of the participants attained longer periods of abstinence (median = 4.5 months) than they had achieved during the preceding three years. More than $90 \%$ of the visit appointments were kept, with only seven patients losing some portion of their deposits. All participants were offered the option of reenlisting in the program after the initial study period. Of the 14 subjects who accepted, more than three fourths remained abstinent for an additional contract period of about six months. Although the financial security deposit and its associated contingencies were thought to be the critical components in the reported success, the investigators allude to the possible significant contribution made by "unspecified social reinforcement" inherent in the program operation. The absence of a control group further increases one's concern about attributing the behavioral outcomes to various aspects of the intervention program.

\section{Drug Abuse}

Promising results were obtained by Boudin ${ }^{24}$ from a case study in which a black female graduate student entered into a three-month contingency contract requiring abstinence from amphetamines and other drugs. The contingency contract was unusual in that: (1) it was witnessed by a third person; (2) it required the subject to check in with the clinic three times each day and to contact the therapist any time that a "potentially dangerous situation" arose (the therapist agreed to be accessible and available 24 hours a day); (3) the subject had to deposit $\$ 500$ in a joint bank account (with the therapist) and agreed to forfeit $\$ 50$ for any actual or suspected drug use; (4) it specified that forfeited funds would be sent to the Ku Klux Klan; and (5) the subject agreed to carry a small shock dispensor with her at all times and to self-administer a shock on any occasion when she felt like obtaining drugs.

During the 12-week study period the subject experienced only one drug-use episode; anecdotal evidence implied that there was no return to amphetamines during a two-year follow-up period. However, the several unique aspects of the contract, the close therapeutic support, and the use of only one study subject make it impossible to assess the singular contribution of the contingency contract.

Hall and associates ${ }^{25}$ applied contingency contracting to the achievement of methadone maintenance by six clients in a treatment program. The subjects were all experiencing serious life difficulties. In this singlesubject reversal design, treatment periods were followed by a noncontingent reinforcement period during which the subject received the average number of credits per week earned during the preceding treatment period, independent of accomplishment of target behavior. Specific contracted behaviors were negotiated with each client and were objectively assessed (eg, urine tests for drug use, time slips for punctual behavior, weekly weighing for weight changes). Only positive reinforcers were used (eg, home delivery of methadone, tickets to special events, reduction in probation time).

Results in the first three to five months showed clearly improved behavior in three of the clients and possible therapeutic effects in two additional subjects. In addition, comparisons of contingent with noncontingent reinforcement periods in two of the three successful cases suggested that the contracted contingencies influenced the behaviors. Follow-up at six and eight 
months revealed that the beneficial outcomes achieved earlier were still present.

Thirty-three subjects (self-referred as well as referred by community agencies or jail) participated in an intensive drug-rehabilitation program that used contingency contracting as a major treatment strategy. Boudin and coworkers ${ }^{26}$ employed primarily paraprofessionals (following six to nine months of training) to develop and implement individualized outpatient programs for each client. Four types of contracts reflecting client progress during the program were used to "shape goal-directed behavior through the extinction of behaviors associated with a drug-dependent lifestyle and replacement of these behaviors with functional antagonists." The "precontract agreement" required each client to relinquish valuable possessions that would be forfeited for failure to follow future contract stipulations. Client responsibilities during this phase included telephoning the clinic frequently, keeping clinic appointments, writing daily logs, providing urine samples, and wearing a behavior counter to record specific drug behaviors.

The "managerial contract" included designated responsibilities for both the client (eg, abstain from drugs, hold a job, keep appointments) and the treatment team (eg, provide personnel for 24-hour crisis intervention, manage joint bank account). Adherence to requirements of the contract led to the clients' earning weekly allowances. Failure by the client to fulfill designated responsibilities resulted in the forfeit of $\$ 5$ in an aversive manner (the money contributed to a cause antithetical to the subject's philosophy); similar noncompliance by the treatment team resulted in the giving of money to the client.

The "transitional contract" allowed for gradual reduction of program structure as the client assumed more responsibility for drug rehabilitation. The final phase of treatment involved the establishment of a "personal contract" detailing self-management of long-range goals. Following completion of the program, clients continued to provide urine samples to the drug project for a follow-up period.

Criteria used to evaluate treatment success included work/school performance, personal/social adjustment, incidence of drug intake, and frequency of arrests or convictions. The minimum criteria established by the investigator for a "positive case outcome" allowed the subjects a negative adjustment rating for only one of the foregoing criteria variables, and any incidence of maladaptive behavior could not be "extreme."

Results represent client involvement in the program ranging from 15 days to 15 months. Positive outcomes were achieved for 13 subjects, negative outcomes for four subjects, and unknown outcomes for two subjects (the remaining 14 subjects were current clients). Furthermore, program completion was significantly positively associated with subjects' years of addiction, the daily cost of the pretreatment drug habit, and the number of times the subject had participated in methadone maintenance programs. However, because the study design contained no control group and because all of the contract interventions were administered to all the clients, it is impossible to draw causal conclusions about either the overall or differential effects of the contracts. Moreover, the variable study period across subjects makes "success" difficult to evaluate (there are no long-term follow-up results per se, although the reporting period for some subjects is considerable).

\section{Renal Disease Regimen}

Patients with end-stage renal disease who receive regular ambulatory hemodialysis treatments must limit their intake of potassium and fluids. Cummings and associate $^{27}$ assessed the usefulness of contingency contracting to increase regimen compliance. Using a randomized control design, four study groups were created: provider-client contract (with or without involvement of a significant other); weekly telephone contact by a nurse (which included verbal reinforcement of any instances of appropriate compliance behaviors); and a nonintervention control (routine care only).

Over the six-week study period, a number of contracts were negotiated between the nurse and the patient; each contract established a timetable for accomplishment of the agreed-upon behaviors. The patient earned points for achievement of contract goals, and these points were converted into rewards (state lottery tickets).

Results after six weeks demonstrated that all three interventions achieved significant reductions in serum potassium levels as compared with the control group; however, the two contract conditions were not more effective than the telephone-contact condition. With regard to limiting fluid intake, the intervention groups again achieved better compliance than did the controls, but the reduction was statistically significant only for those patients whose contract included a significant other. Follow-up at three months revealed that all of the earlier intervention effects had disappeared.

Another attempt to implement contingency contracting in the area of renal disease is reported in two case studies undertaken by Keane and associates. ${ }^{28}$ After assessment of baseline intersession weight gain, contingency contracts were negotiated between each subject and staff; for meeting the reduced weight gain criterion, one subject would be rewarded with early-morning dialysis sessions, while the other subject would receive preferred meals. The overall behavioral treatment program also included such social reinforcers as praise from and social interaction with the medical staff for meeting the weight gain criterion. One patient was also asked to graph her intersession weight gain. 
The study design for one subject included a second (postcontract) baseline period (32 sessions), which did not involve any contingencies for retaining the morning dialysis sessions, followed by a second contract wherein retaining the morning schedule was dependent upon achieving the reduced weight gain goal (a "punishment" procedure). A similar procedure was also followed for the other subject (baseline plus initial contract); however, this was followed directly by a second contract, and involvement with a psychologist was gradually reduced.

Findings indicated substantial improvement in regimen adherence by both subjects during the contracting intervention periods. While no data are provided on long-term outcomes, the authors state that "continuous follow-up assessment indicated long-term maintenance of treatment effects for these patients." The relative contribution of contingency contracting to these study results is again difficult to determine owing to the lack of a control group, the presence of a behavioral program including reinforcers beyond those stipulated by the contract, and the small number of subjects involved.

\section{Antihypertensive Regimen}

Patient noncompliance with antihypertensive therapy is notoriously high because of the generally asymptomatic nature of the condition and the complexity, duration, and side effects of treatment. To assess the ability of contingency contracts to ameliorate the problem, Swain and Steckel ${ }^{29}$ used an experimental design in which adult hypertensive outpatients were randomly assigned to one of three groups: group A, a control group receiving routine care; group B, routine care plus patient education (five instructional booklets and subsequent opportunity to ask questions of the research nurse); and group $C$, routine care, patient education, plus a contingency contract between the patient and nurse that specified health goals and contingent rewards. All subjects completed baseline tests of knowledge.

With regard to contracted behaviors, all group $\mathrm{C}$ subjects specified a target posttest score and an associated reward. Additional behaviors identified by patients as ones they wished to begin working on were included in subsequent contracts (eg, weight loss, keeping clinic appointments, diet management). Patients also set their own rewards, resulting in considerable variation (eg, lottery tickets, books, magazines, additional time with a health-care provider, assistance in completing insurance forms).

After 18 months (four clinic visits), group A exhibited considerable fluctuation in diastolic blood pressure and ranked second in clinic dropout (no knowledge posttest was obtained). Group B also exhibited substantial variations in diastolic blood pressure, ranked highest in dropouts, and scored second in the knowledge posttest. In group $\mathrm{C}$, diastolic blood pressure fell (by the second visit) below the clinic's standard for achievement of control and remained at that level; there were no dropouts, and these subjects achieved the highest posttest knowledge score. The authors further state that none of the group $\mathrm{C}$ patients failed to abide by their contracts.

Because the contracting intervention was implemented only in combination with patient education, it is difficult to evaluate its independent contribution. Also the absence of a knowledge posttest for group A prevents assessment of the possible influence on knowledge exerted by the pretest experience.

\section{DISCUSSION}

The 15 studies reviewed demonstrate at least short-term positive effects from contingency contracting. This intervention strategy appears to have been beneficial across a variety of medical conditions and health-related behaviors and for both inpatient and outpatient settings. Furthermore, investigators who have used contracts generally report that they are relatively simple to implement, can be employed by paraprofessionals, and do not add much time to the provider-client interaction. However, these studies have a number of conceptual and methodological difficulties that hinder interpretation of their findings.

\section{Problems of Design}

Only seven of the 15 investigations randomly assigned participants to study conditions, and only five used control groups. Moreover, the contingency contracting element is often embedded in a more elaborate behavioral treatment program, often preventing clear attribution of outcomes to the contract alone. Six studies lacked long-term follow-up. Where long-term results were available, they evidenced considerable recidivism (except where the contracting process was continued throughout the follow-up period). This suggests that contingency contracting frequently shares with other behavioral treatment approaches the problem of not being combined with other strategies that influence compliance behavior, such as improved and continued provider-client relationship, social support, and modified health attitudes and beliefs.

\section{Problems of Generalizability}

Most of these studies were conducted with motivated volunteers rather than random samples of some defined population. In many cases, substantial numbers of potential subjects declined to participate after study requirements were described. This rejection phenomenon raises many questions, specifically about the interpretation of study results and, more generally, about the appeal of contingency contracts to clients. Perhaps a substantial portion of this high refusal rate can be explained by the requirement (in most studies) of client 
forfeiture of money or other valued items as the contracted contingency.

In general, the investigations were conducted with small numbers of subjects; of the 15 studies reviewed, only two had more than 50 participants, and eight had 25 or less. This small-sample problem was frequently exacerbated by subject dropout during the study period.

\section{Problems of Contract Design and Process}

In an earlier section of this article we enumerated the components of a "good" contingency contract-components specified by leading theorists and investigators in the contracting field. Unfortunately, many of these critical elements are, in the studies reviewed here, conspicuous by their absence. For example, the behaviors and reinforcers were seldom negotiated; provider responsibilities were often poorly specified; positive reinforcers (other than return of clients' own valuables) were infrequently used; the client was rarely weaned from the intervention strategy; and reinforcement was often provided at fixed intervals (as opposed to coming directly after the desired behavior).

Some studies relied on a single contracting instrument, while others employed multiple contracts over time, and the value of the reinforcer varied enormously across studies (seemingly uncorrelated with the effort required by the behavior). These disparities between ideal and real contract design and process may account for the limited results achieved in many studies.

\section{CONCLUSION}

Contingency contracting appears to be a useful addition to the repertoire of compliance-enhancing strategies. The contract formally elaborates regimen expectations and establishes client commitment to achieving treatment goals. The contracting process also helps to combat the stereotype of the ideal client as a passive recipient of medical care. This approach appears beneficial for increasing adherence to short-term regimens and for getting clients started on long-term therapies.
However, while existing studies represent important steps toward evaluating the contingency contracting process, difficulties in subject selection, design, and implementation make assessment of their results rather tentative. To further refine our understanding of the potential of contracting, additional investigation must address: (1) the types of clients and providers for which this strategy is most appealing and effective (eg, will some providers feel less spontaneous and powerful if asked to follow a fixed, systematic program?); (2) the skills that providers must acquire in order to implement contingency contracting; (3) the power of contracts that conform more closely to "ideal" contracting criteria; (4) the mechanisms for continued contracting or for weaning the client from contingency dependence; (5) the circumstances under which long-term results might be obtained; and (6) the effectiveness of contracting when used in combination with other strategies to increase client cooperation. In addition, studies have (with few exceptions) used reinforcements that seem to have little applicability and relevance to the realities of the structure of the health-care delivery system. In many cases, clients had to initially relinquish considerable sums or other valuables or were provided with rewards that exceed the system's normal capacity to implement, particularly on a large scale. Further study is therefore needed to develop creative ways of transferring the contingency element of prior research to the real world of clinical practice.

It has been posited that the best type of contract is a "self-contract," or one in which the person administers his/her own reward and another person is available only for support and, possibly, advice. ${ }^{30}$ This type of contract not only is less contrived and less costly than patient-provider contingency contracts, but also it can serve to enhance the self-control skills of the individual. Self-contracts may also prove valuable as a method of moving the patient away from the dependence created by the initial provider-client contract. Unfortunately, no controlled studies to date have examined the effectiveness of self-contracts.

\section{REFERENCES}

1. Sackett DL. The magnitude of compliance and noncompliance. In: Sackett DL, Haynes RB, eds. Compliance with therapeutic regimens. Baltimore, Maryland: The Johns Hopkins University Press, 1976:26-39.

2. Dunbar JM, Stunkard AJ. Adherence to diet and drug regimen. In: Levy R, Dennis BH, Rifkind BM, Ernst N, eds. Nutrition, lipids and coronary heart disease. New York: Raven Press, 1979:391-423

3. Lewis $\mathbf{C E}$, Michnich $\mathbf{M}$. Contracts as a means of improving patient compliance. In: Barofsky I, ed. Medication compliance: A

behavioral management approach. Thorofare, New Jersey: Charles B. Slack, Inc, 1977:69-75.

4. Skinner BF. Science and human behavior. New York: MacMillan, 1953.

5. Pomerleau O, Bass F, Crown V. Role of behavior modification in preventive medicine. $N$ Engl J Med 1975; 292:1277-1282.

6. Sulzer ES. Reinforcement and the therapeutic contract. $J$ Couns Psychol 1962; 3:109-112.

7. Homme LE. Contiguity theory and contingency management. Psychol Record 1966; 16:223-241.

8. Homme LE, Csanyi M, Gonzales J, Rechs J. How to use contingency contracting in the classroom. Champaign, Illinois: Research Press, 1969. 
9. Etzwiler DD. The contract for health care. J Am Med Assoc 1973; $224: 1034$.

10. Kanfer FH. The many faces of self-control or behavior modification changes its focus. In: Stuart RB, ed. Behavioral selfmanagement. New York: Brunner/Mazel, 1977:1-49.

11. Stuart RB. Behavior contracting with families of delinquents. $J$ Behav Ther Exp Psychiatr 1971; 2:1-11.

12. Kanfer FH. Self-management methods. In: Kanfer FH, Goldstein AP, eds. Helping people change. New York: Pergamon Press, 1980:349-353.

13. Steckel SB. Patient contracting. Norwalk, Connecticut: Appleton-Century-Crofts, 1982.

14. Melamed BG, Siegel LJ. Behavioral medicine: Practical applica tions in health care. New York: Springer Publishing Co, 1980:3-92.

15. Harris MB, Bruner CG. A comparison of a self-control and a contract procedure for weight control. Behav Res Ther 1971; 9:347-354.

16. Aragona J, Cassady J, Drabman RS. Treating overweight children through parental training and contingency contracting. $J$ Appl Behav Anal 1975; 8:269-278.

17. Mann RA. The behavior-therapeutic use of contingency contracting to control an adult behavior problem: Weight control. In: Katz RC, Zlutnick S, eds. Behavior therapy and health care: Principles and applications. New York: Pergamon Press, 1975:567.583.

18. Agras S, Werne J. Behavior modification in anorexia nervosa: Research foundations. In: Vigersky RA, ed. Anorexia nervosa. New York: Raven Press, 1977:291-303.

19. Jeffery RW, Thompson PD, Wing RR. Effects on weight reduction of strong monetary contracts for calorie restriction or weight loss. Behav Res Ther 1978; 16:363-369.
20. Elliott R, Tighe T. Breaking the cigarette habit: Effects of a technique involving threatened loss of money. Psychol Record $1968 ; 18: 503-513$.

21. Winett RA. Parameters of deposit contracts in the modification of smoking. Psychol Record 1973; 23:49-60.

22. Miller PM, Hersen M, Eisler RM. Relative effectiveness of instructions, agreements, and reinforcement in behavioral contracts with alcoholics. J Abnorm Psychol 1974; 83:548-553.

23. Bigelow G, Strickler D, Liebson I, Griffiths R. Maintaining disulfiram ingestion among outpatient alcoholics: A securitydeposit contingency contracting procedure. Behav Res Ther 1976; 14:378-381.

24. Boudin HM. Contingency contracting as a therapeutic tool in the deceleration of amphetamine use. Behav Ther 1972; 3:604-608.

25. Hall SM, Cooper JL, Burmaster S, Polk A. Contingency contracting as a therapeutic tool with methadone maintenance clients: Six single subjects studies. Behav Res Ther 1977; 15:438-441.

26. Boudin HM, Valentine VE, Inghram RD, et al. Contingency contracting with drug abusers in the natural environment. Int $J$ Addict 1977; 12:1-16.

27. Cummings KM, Becker MH, Kirscht JP, Levin NW. Intervention strategies to improve compliance with medical regimens by ambulatory hemodialysis patients. J Behav Med 1981; 4:111-127.

28. Keane TM, Prue DM, Collins FL Jr. Behavioral contracting to improve dietary compliance in chronic renal dialysis patients. $J$ Behav Ther Exp Psychiatr 1981; 12:63-67.

29. Swain MA, Steckel SB. Influencing adherence among hypertensives. Res Nurs Health 1981; 4:213-222.

30. DiMatteo MR, DiNicola DD. Achieving patient compliance: The psychology of the medical practitioner's role. New York: Pergamon Press Inc, 1982.

\section{APPENDIX}

\section{Date \\ HEALTH-CARE CONTRACT}

Contract Goal: (Specific outcome to be attained)

I, (client's name), agree to (detailed description of required behaviors, time and frequency limitations)

in return for (positive reinforcements contingent upon completion of required behaviors; timing and mode of delivery of reinforcements)

1, (provider's name), agree to (detailed description of required behaviors, time and frequency limitations)

(Optional) I, (significant other's name), agree to (detailed description of required behaviors, time and frequency limitations)
(Optional) Aversive consequences: (Negative reinforcements for failure to meet minimum behavioral requirements)

(Optional) Bonuses: (Additional positive reinforcements for exceeding minimum contract requirements)

We will review the terms of this agreement, and will make any desired modifications, on (date). We hereby agree to abide by the terms of the contract described above.

Signed: (Client)

Signed: (Significant other, if relevant)

Signed: (Provider)

Contract effective from (Date)

to (Date) 